\title{
Cigarrillo electrónico: ¿otra herramienta para ayudar a dejar de fumar o una nueva estrategia para promover la adicción a la nicotina?
}

Malen Cubas ${ }^{\ddagger}$, Gabriela Deluca Bisurgi ${ }^{\ddagger}$, Mercedes Seeber ${ }^{\ddagger}$, Silvina Sffaei ${ }^{\ddagger}$, Agustina Maturis y Sergio Terrasa**

\section{Resumen}

Los autores de este artículo describen las principales características del cigarrillo electrónico y sus componentes, analizan la controversia sobre su potencial uso como terapia de sustitución del tabaco y describen las experiencias regulatorias de Argentina y de otros países.

\section{Abstract}

The authors of this article describe the main features of electronic cigarettes and its components, analyze the controversy about its potential use as tobacco substitution therapy, and summarize the regulatory experiences in Argentina and other countries.

Cubas M, Deluca Bisurgi G, Seeber M, Sffaeir S, Maturi A y Terrasa S. Cigarrillo electrónico: ¿otra herramienta para ayudar a dejar de fumar o una nueva estrategia para promover la adicción a la nicotina? Evid Actual Prác Ambul 20180;21(4):94-97.

\section{Tabaquismo en Argentina}

El descenso de la prevalencia del tabaquismo ha sido constante en Argentina: $39,8 \%$ de los adultos en $1999,33,4 \%$ en 2005 , $30,1 \%$ en 2009 , y $29,6 \%$ en varones y $22,3 \%$ en mujeres en $2012^{1}$.

El 14 de Junio de 2011 se promulgó en Argentina la Ley Nacional de Control de Tabaco (№ 26.687) ${ }^{2}$, que estableció las bases para el comienzo de su erradicación. A partir de su promulgación se prohibió el consumo de tabaco en espacios cerrados públicos y privados, su venta a menores de 18 años, la venta de cigarrillos sueltos, y la publicidad, promoción y patrocinio de cigarrillos en medios de comunicación o vía pública, estableciéndose la obligación de que los paquetes incluyan imágenes con advertencias sanitarias.

Es en este contexto de avance de las medidas antitabáquicas a nivel mundial que la industria tabacalera comenzó a explorar nuevas opciones para mantener su negocio.

\section{El cigarrillo electrónico}

Durante años se ha intentado producir un producto que reemplace al cigarrillo tradicional, pero sin sus efectos nocivos. En
1963 el estadounidense Herbert Gilbert creó el primer prototipo de cigarrillo electrónico $(\mathrm{CE})^{3}$, pero por sus limitaciones tecnológicas, éste nunca llegó a distribuirse. Fue en 2003 cuando el primer CE fue introducido en el mercado de la mano del farmacéutico chino Hon $\mathrm{Lik}^{4}$, quien lo patentó por primera vez, comenzando al año siguiente su fabricación masiva y su distribución mundial.

Este producto consiste en un dispositivo con forma similar al cigarrillo convencional, provisto de un mecanismo microelectrónico con una batería recargable, que vaporiza cartuchos que pueden contener cantidades variables de nicotina (0 a $24 \mathrm{mg}$ ), aceites esenciales de tabaco, componentes aromatizantes, propilenglicol, glicerol y otras sustancias ${ }^{5}$. Tratando de imitar a los cigarrillos tradicionales, el CE produce un vapor visible que es inhalado al momento de ser fumado y también es emitido al ambiente circundante ${ }^{6}$.

En forma análoga a lo que había ocurrido en décadas pasadas, cuando el tabaco no era considerado dañino, durante los últimos años ha crecido la evidencia de que el cigarrillo electrónico (CE) no es tan inocuo como se creía ${ }^{7}$. Sus principales componentes se describen en la tabla 1.

Tabla 1: principales componentes del cigarrillo electrónico.

\begin{tabular}{l|l}
\multicolumn{1}{c|}{ Componente } & \\
\hline Propilenglicol & $\begin{array}{l}\text { Constituye la mayor parte del líquido que se utiliza para vaporizar y es el responsable de que el vapor sea visible. Si bien la Administración de } \\
\text { Alimentos y Medicamentos de EEUU (en inglés FDA) ha determinado que el propilenglicol es "...generalmente considerado como seguro para uso en } \\
\text { alimentación, cosmética, y medicinas..."; su consumo inhalatorio produce a corto plazo irritación de los ojos, garganta y vía respiratoria, y a largo } \\
\text { plazo, asma en los niños. }\end{array}$ \\
\hline Glicerina & $\begin{array}{l}\text { De origen vegetal, se utiliza como base para el líquido del CE. El efecto que se ha podido estudiar hasta el momento es la aparición de nuevos casos } \\
\text { de neumonía lipoidea relacionada con el vapor. }\end{array}$ \\
\hline Nicotina & $\begin{array}{l}\text { Con efectos sobre el sistema nervioso central, es una de las sustancias psicoactivas más adictivas que existen. Al inhalar, el humo hace llegar la } \\
\text { nicotina a los pulmones, que de allí pasa a la sangre por medio de los alvéolos y diez a sesenta segundos después, atraviesa la barrera } \\
\text { hemato-encefálica penetrando en el cerebro. La nicotina es la responsable de la dependencia. Es importante remarcar que aquellos ex fumadores } \\
\text { que utilizan el CE, mantienen su dependencia al tabaco. }\end{array}$ \\
\hline Dietilenglicol & Es una sustancia tóxica incluida como propelente, utilizada también como anticongelante de autos y con evidencia de potencial efecto carcinogénico. \\
\hline Formaldehido, acetaldehído & Son citotóxicos, cancerígenos, irritantes y causantes de enfisema pulmonar y dermatitis. \\
\hline y acroleína & Carcinógeno, neurotóxico e irritante. \\
\hline Molueno & El cromo, el níquel, el zinc y el plomo son hepatotóxicos y nefrotóxicos. \\
\hline
\end{tabular}

‡ Escuela de Medicina del Instituto Universitario Hospital Italiano de Buenos Aires.

$\S$ Servicio de Medicina Familiar y Comunitaria del Hospital Italiano de Buenos Aires.

** Departamento de Salud Pública del Instituto Universitario Hospital Italiano y Servicio de Medicina Familiar y Comunitaria del Hospital Italiano de Buenos Aires sergio.terrasa@ hiba.org.ar. 
Los CE generan niveles séricos de cotinina (metabolito de la nicotina) similares al cigarrillo fumado $(60,6 \pm 34,3$ versus 61,3 $\pm 36,6 \mathrm{ng} / \mathrm{mL}$ respectivamente) ${ }^{8}$ y han sido diseñados para ser utilizados en reemplazo de los cigarrillos convencionales en los lugares donde se prohíbe fumar. Sin embargo, algunos de sus productores los proponen como una estrategia para dejar de fumar, aduciendo además que están desprovistos de los efectos deletéreos de los productos de la combustión del tabaco.

Por ejemplo y como afirma la disposición 3.226 de la Administración Nacional de Medicamentos, Alimentos y Tecnología Médica (ANMAT) de la República Argentina ${ }^{9}$, algunas publicidades en internet promocionan estos dispositivos dispensadores de nicotina como “... productos creados para mejorar la calidad de vida con sabor a cigarrillo y vapor simulador del humo, pudiendo ser usados en cualquier lugar ya que no tienen olor ni dañan la salud...".

Sin embargo y como hemos descripto previamente, la utilización de estos productos también genera emisión de propilenglicol, nicotina y sustancias cancerígenas, que pueden contaminar los espacios cerrados, con los consecuentes riesgos para quienes están expuestos en forma pasiva a su inhalación.

\section{Evidencia sobre los riesgos del cigarrillo electrónico}

Luego de identificar 1.101 investigaciones originales en las bases de datos PubMed, EMBASE y CINAHL, Pisinger y col. incluyeron en su revisión sistemática 76 estudios que habían investigado los efectos de los fluidos y/o los vapores del CE sobre la salud humana o sobre modelos animales experimentales, muchos de ellos con muy pocas observaciones e importantes problemas metodológicos. Además, en $34 \%$ de estas publicaciones, los autores identificaron importantes conflictos de intereses. Entre los efectos comunicados por los estudios originales se destacan la citotoxicidad $^{\dagger \dagger}$ y los cambios de expresiones genéticas. Por otro lado, estudios experimentales documentaron incrementos en la resistencia de las vías aéreas luego de mínimas exposiciones, concluyendo Pisinger y col. ${ }^{10}$ que por ahora no ha podido documentarse la seguridad de los CE.

\section{Controversia sobre el potencial uso del cigarrillo electrónico como terapia de sustitución del tabaco}

El ingreso del CE al mercado es sumamente reciente, por lo tanto la calidad y la cantidad de su composición se encuentran en constante evolución. Sin embargo cabe hacernos la siguiente pregunta: ¿Podemos considerar al cigarrillo electrónico como un dispositivo que ayudará a reducir el consumo de tabaco, o simplemente se trata de una nueva estrategia de marketing de las grandes empresas para atraer al "nuevo fumador", tratando de enmascarar los riesgos de su consumo? Los autores de este artículo nos inclinamos por la última de las opciones.

El Grupo de Estudio de la Organización Mundial de la Salud (OMS) sobre la Reglamentación de los Productos de Tabaco se expidió en 2008 respecto del CE, sosteniendo que "...no se ha establecido la seguridad y el grado de absorción de la nicotina a través de estos productos...". En el mismo documento menciona que actualmente, la evidencia es insuficiente para concluir que los CE sean una ayuda eficaz para dejar de fumar o que liberen nicotina en dosis adecuadas como para ser utilizados en la deshabituación tabáquica, no existiendo pruebas suficientes que determinen que sean seguros para el consumo humano. También sostienen que el uso de los CE -en lugar de tratamientos basados en evidencia científica- para dejar de fumar, podría retrasar la cesación tabáquica en el caso de que estos productos no sean eficaces ${ }^{4}$.

Por lo mencionado previamente y por la falta de más investigaciones al respecto, es que desde 2013 la OMS viene desaconsejando hasta ahora el uso de dispositivos de liberación de nicotina como los $\mathrm{CE}^{3}$.

Sin embargo, existe incipiente evidencia clínica de la eficacia del CE en cesación tabáquica que vale la pena tener en cuenta. Por esta razón resumimos a continuación dos revisiones sistemáticas publicadas en 2016 y un ensayo clínico publicado en 2019.

Con el objetivo de evaluar la seguridad y la efectividad del CE para ayudar a las personas que fuman a lograr abstinencia a largo plazo, Hartmann-Boyce $\mathrm{J}$ y col. ${ }^{11}$ revisaron los artículos publicados entre 2004 y 2016 indizados en el Registro especializado del grupo Cochrane de adicción al tabaco (Cochrane Tobacco Addiction Group) y en el Registro Cochrane Central de Ensayos Controlados (CENTRAL), MEDLINE, Embase y PsycINFO, verificando además las referencias y contactando a los autores de cada una de las publicaciones originales. Incluyeron finalmente dos estudios, con un total de 957 pacientes, realizados en Nueva Zelanda e Italia, cuyos resultados se resumen en la tabla 2.

Tabla 2: eficacia relativa del cigarrillo electrónico (evaluada a través del monóxido de carbono exhalado), comparada con cigarrillo electrónico "placebo" y con terapias aprobadas de reemplazo de nicotina.

\begin{tabular}{c|c|c|c} 
Grupo de comparación & Seguimiento & $\begin{array}{c}\text { Riesgo relativo } \\
\text { (IG 95\%) }\end{array}$ & $\begin{array}{c}\text { Calidad de la evidencia } \\
\text { (GRADE) }\end{array}$ \\
\hline $\begin{array}{c}\text { CE "placebo" } \\
\mathbf{n} \text { total: } \mathbf{6 6 2} \text { (2 estudios) }\end{array}$ & 6 a 12 meses & $2,29(1,05$ a 4,96) & BAJA \\
\hline TNAC & 6 meses & $1,26(0,68$ a 2,34) & $\oplus \oplus \ominus \ominus$ \\
\hline $\mathbf{n}$ total: 584 (1 estudio) & & & MUY BAJA \\
\hline
\end{tabular}

CE: cigarrillo electrónico. TNAC: Terapia de nicotina aprobada para la cesación tabáquica. IC: intervalo de confianza.

\footnotetext{
t† Ha sido estudiado el comportamiento de los fluidos de recarga de los CE frente a tres tipos de células: los fibroblastos de los pulmones humanos, las células madres de los embriones humanos y las células madre del sistema neural de los ratones. Si bien no se pudo documentar citotoxicidad sobre los fibroblastos pulmonares, se observaron efectos citotóxicos sobre ambos tipos de células madre (las de ratón y las de embriones humanos), concluyéndose que este daño habría sido causado por los aromatizantes utilizados.
} 
Destacamos además los resultados de la revisión sistemática publicada por Khalkoran y col. ${ }^{12}$ que tuvo un carácter más pragmático ya que además de los dos ensayos clínicos que había incluido la de Hartmann-Boyce $\mathrm{J}$ y col. ${ }^{11}$, incluyó 36 estudios observacionales. Estos autores documentaron que el uso de CE se asoció a una probabilidad casi $30 \%$ menor de lograr dejar de fumar (OR 0,72; IC95\% 0,57 a 0,91) que el no uso de estos dispositivos.

Por otro lado, si bien no superan la eficacia de la mayoría de las terapias de reemplazo nicotínico actualmente autorizadas en la mayoría de los países, destacamos los resultados del ensayo clínico recientemente publicado en enero de 2019 por Hajek $P$ y col. ${ }^{13}$ (resumido y comentado en este número de EVIDENCIA), que documentó una tasa de abstinencia al año de $18,0 \%$ en el grupo CE vs un $9,9 \%$ en el grupo que recibió parches, sprays, chicles y/o tabletas de nicotina (RR: 1,83; IC $95 \% 1,30$ a 2,58; $\mathrm{p}<0,001)$. Sin embargo, el $80 \%$ de las personas asignadas a CE contunuaba usándolo al año vs el $9 \%$ de los asignados al grupo control.

\section{Ejemplos a nivel mundial de la legislación sobre el cigarrillo electrónico}

Las leyes sobres los CE en todo el mundo cambian continuamente por lo mencionado en párrafos anteriores (modificaciones que se realizan sobre los CE en cuanto a sus componentes y concentraciones, nuevos trabajos de investigación que van documentando esos sucesos). Sin embargo, y dependiendo de los diferentes objetivos regulatorios, las leyes relevantes en el mundo se pueden dividir en las siguientes categorías principales:

1. Prohibición de todas las actividades vinculadas al CE (venta, importación, fabricación y consumo): Brasil ${ }^{\ddagger}$, Canadá, Colombia, Hong Kong§§, México, Nueva Zelanda, Singapur, Tailandia***, Taiwán y Uruguay ${ }^{\dagger+\dagger} 8$.

2. Prohibición de la venta pero no de la posesión de CE (Australia ${ }^{\ddagger \ddagger \ddagger)}$.

3. Contemplación del CE como una terapia de sustitución: EE.UUß\$s y UK ${ }^{14,15}$.

\section{La toma de posición de los organismos regulatorios de Ar- gentina}

En consonancia con la toma de posición de la OMS, la ANMAT comunicó que: a) a corto plazo, se han hallado efectos fisiológicos adversos en las vías respiratorias similares a aquellos asociados al humo del tabaco, requiriéndose más estudios para conocer los efectos a largo plazo; b) se han encontrado sustancias cancerígenas en los líquidos y el vapor de los cigarrillos electrónicos; c) se han descrito numerosas intoxicaciones y efectos adversos (algunos severos) relacionados con estos productos.

Por otro lado, la disposición 3.226/2011 publicada el 9 de mayo del 2011 en el boletín oficial ${ }^{9}$ es bien clara al respecto: “... prohíbese la importación, distribución, comercialización y la publicidad o cualquier modalidad de promoción en todo el territorio nacional de administración de nicotina denominado cigarrillo electrónico..."

En 2016, la ANMAT ratificó la decisión comunicada en la disposición $3.226 / 11^{16}$ de prohibir la importación, distribución, comercialización y la publicidad o cualquier modalidad de promoción del cigarrillo electrónico en todo el territorio nacional, debido a la escasa evidencia sobre la eficacia y seguridad a largo plazo del cigarrillo electrónico.

\section{Reflexiones finales}

El CE tiene una corta vida en el mercado y está en constante evolución (tanto en sus componentes como en las concentraciones de estos), lo que dificulta todavía que podamos arribar a conclusiones concretas, ya que sus potenciales efectos nocivos a largo plazo siguen en revisión. Sin embargo, como ya fue mencionado anteriormente, el CE posee tanto componentes adictivos como la nicotina, como componentes cancerígenos que demostraron tener efectos citotóxicos sobre células madre de ratones y células de embriones humanos.

En Argentina el CE continúa prohibido por ser considerado un método cuya seguridad y eficacia todavía están en discusión.

Los autores de esta revisión consideramos que no contamos con evidencia suficiente respecto de la seguridad y eficacia del CE como para recomendarlo como terapia de sustitución del tabaco en pacientes que desean dejar de fumar. A nuestro entender, quedaría reservado su uso solo como una estrategia de minimización de daños en personas que no hayan podido abandonar el tabaquismo luego de intentos frustrados en los que hayan empleado otras herramientas de probada eficacia, recomendándoles también que no vapeen $C E$ en espacios cerrados.

Agradecemos a los Dres Javier Saimovici y Guillermo Espinosa por la bibliografía aportada y los consejos para mejorar este artículo. Este documento cuenta con el aval de la Asociación Metropolitana de Medicna Familiar y la Asociación Argentina de Tabacología.

执 Si bien en Brasil no existe una legislación no tan clara sobre los CE, se prohibió su fabricación y su venta desde 2014.

$\$ \S$ En Hong Kong está prohibida la posesión y la venta de e-líquidos con nicotina. Al ser este producto tipificado legalmente como un veneno, llevar una botella con este está castigado por una pena de cárcel de dos años.

${ }^{* \star \star}$ En Tailandia el CE fue prohibido en 2014. Fumar CE en la calle puede ser penalizado y hasta puede ser motivo de encarcelamiento.

ttt Uruguay impuso una prohibición estricta hacia los cigarrillos electrónicos en 2009.

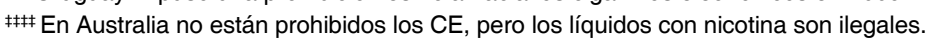

${ }^{\$ \S \S}$ En EEUU la regulación del CE es similar a la del tabaco. 


\section{Referencias}

1. Organización Panamericana de la Salud. U.S. Center of Disease Control. Instituto Nacional de Estadísticas y Censo de Argentina. Ministerio de Salud. Presidencia de la Nación. Encuesta Mundial de Tabaquismo en Adultos. Argentina 2012. 30 de Mayo de 2013. Buenos Aires, Argentina. ISBN N978-950-38-0143-7 Disponible en URL: http://www. msal.gob.ar/tabaco/images/stories/info-equipos-de-salud/pdf/2013-09 encuesta-tabaquismo-adultos-25-junio.pdf (último acceso 08/02/2019).

2. Argentina tiene ya una ley nacional de control del tabaco. En: Programa Nacional de Control del Tabaco. Secretaría de Gobierno de Salud. Ministerio de Salud y Desarrollo Social. Presidencia de la Nación. Disponible en URL: http://www.msal.gob.ar/tabaco/index.php/component/content/article/4-destacados-slide/82-argentina-tiene-una-ley-nacionalde-control-de-tabaco (último acceso 08/02/2019)

3. Gilbert H. Smokeless non-tobacco cigarette. 3.200.819 Patented Aug 17, 1965, Ser. No. 273,624 10 Caimas. (C. 128-208). Disponible en URL: https://patentimages.storage. googleapis.com/a8/a4/ef/28cea2ddb9f425/US3200819.pdf (último acceso 08/02/2019).

4. Nayir E y col. Electronic cigarette (e-cigarette). Journal of Oncological Science 2 (2016) 16-20. Disponible en URL: https://ac.els-cdn.com/S2452336416300188/1-s2.0S2452336416300188-main pdf? tid=dd6c225b-733b-4a66-aefe-e503d67b9e22\&acdnat=1549657546 d41dd833e1c12dd4217f52b332f019f7 (último acceso 08/02/2019).

5. National Academies of Sciences, Engineering \& Medicine (U.S.). Committee on the Review of the Health Effects of Electronic Nicotine Delivery Systems. Public Health Consequences of E-cigarettes: A Consesus Study Report of the National Academies of Sciences, Health, Medicine. (2018).

6. Zainol Abidin N y col. Electronic cigarettes and indoor air quality: a review of studies using human volunteers. Rev. Environ. Health 32, 235-244 (2017).

7. Hua M y col. Potential health effects of electronic cigarettes: A systematic review of case reports. Preventive Medicine Reports 4 (2016) 169-178. Disponible en URL: https:// www.clinicalkey.com/service/content/pdf/watermarked/1-s2.0-S2211335516300523.pdf?locale=en_US (último acceso 08/02/2019).

8. Argentina. Ministerio de Salud de la Nación Guía rápida e-cig. - 1a ed. - Ciudad Autónoma de Buenos Aires : Ministerio de Salud de la Nación, 2017.14 p. ; 22 x 15 cm. ISBN 978-950-38-0247-2 1. Acceso a la Salud. 2. Neumonología. I. Título. CDD 614.592. Disponible en URL: http://www.msal.gob.ar/images/stories/bes/graficos/0000000973c nt-2018-01-22 guia-rapida-cigarrillo-electronico.pdf (último acceso 12/02/2019).

9. Administracion Nacional de Medicamentos, Alimentos y Tecnología Médica. Disposición 3226/2011. Prohíbese la comercialización y uso en todo el territorio nacional de un determinado producto. Bs. As., 6/5/2011. Disponible en URL: http://www.anmat.gov.ar/webanmat/retiros/mayo/Disposicion_3226-2011.pdf (último acceso 08/02/2019).

10. Pisinger C. A systematic review of health effects of electronic cigarettes. Preventive Medicine 69 (2014) 248-260. Disponible en URL: https://ac.els-cdn.com/ S0091743514003739/1-s2.0-S0091743514003739-main.pdf?_tid=1ac082f9-1a79-412f-80ce-ab3a92405a59\&acdnat=1549656518_1e1980325cb5a6633936d0ced7aa692e (último acceso 08/02/2019).

11. Hartmann-Boyce J y col. Electronic cigarettes for smoking cessation (Review). Cochrane Database of Systematic Reviews 2016, Issue 9. Art. No.: CD010216

12. Kalkhoran S, Glantz SA. E-cigarettes and smoking cessation in real-world and clinical settings: a systematic review and meta-analysis. The Lancet Respiratory Medicine. 2016. Feb;4(2):116-28. Disponible en URL: https://www.ncbi.nlm.nih.gov/pmc/articles/PMC4752870/ (último acceso 06/03/2019).

13. Hajek $\mathrm{P}$ y col. A randomized trial of e-cigarettes versus nicotine-replacement therapy. N Engl J Med. DOI: 10.1056/NEJMoa1808779.

14. National Health Service (UK). Using e-cigarettes / vapes to quit smoking. Disponible en URL: https://www.nhs.uk/oneyou/for-your-body/quit-smoking/using-e-cigarettes-vapesto-quit-smoking (último acceso 12/02/2019).

15. Tubella P y Torrez Menárquez A. Reino Unido promueve el vapeo para dejar de fumar. La OMS y varias sociedades científicas mantienen que es dañino. Diario El País. Disponible en: https://elpais.com/sociedad/2018/12/28/actualidad/1545988444_052820.html (último acceso 12/02/2019).

16. Agencia Nacional de Medicamentos Alimentos y Tecnologías Médicas. Seguridad y eficacia del cigarrillo electrónico en la práctica clínica. Informe de evaluación de tecnología sanitaria. 2016. Disponible en URL: http://www.anmat.gov.ar/ets/INFORME_CE_20-12-2016.pdf (último acceso 12/02/2019)

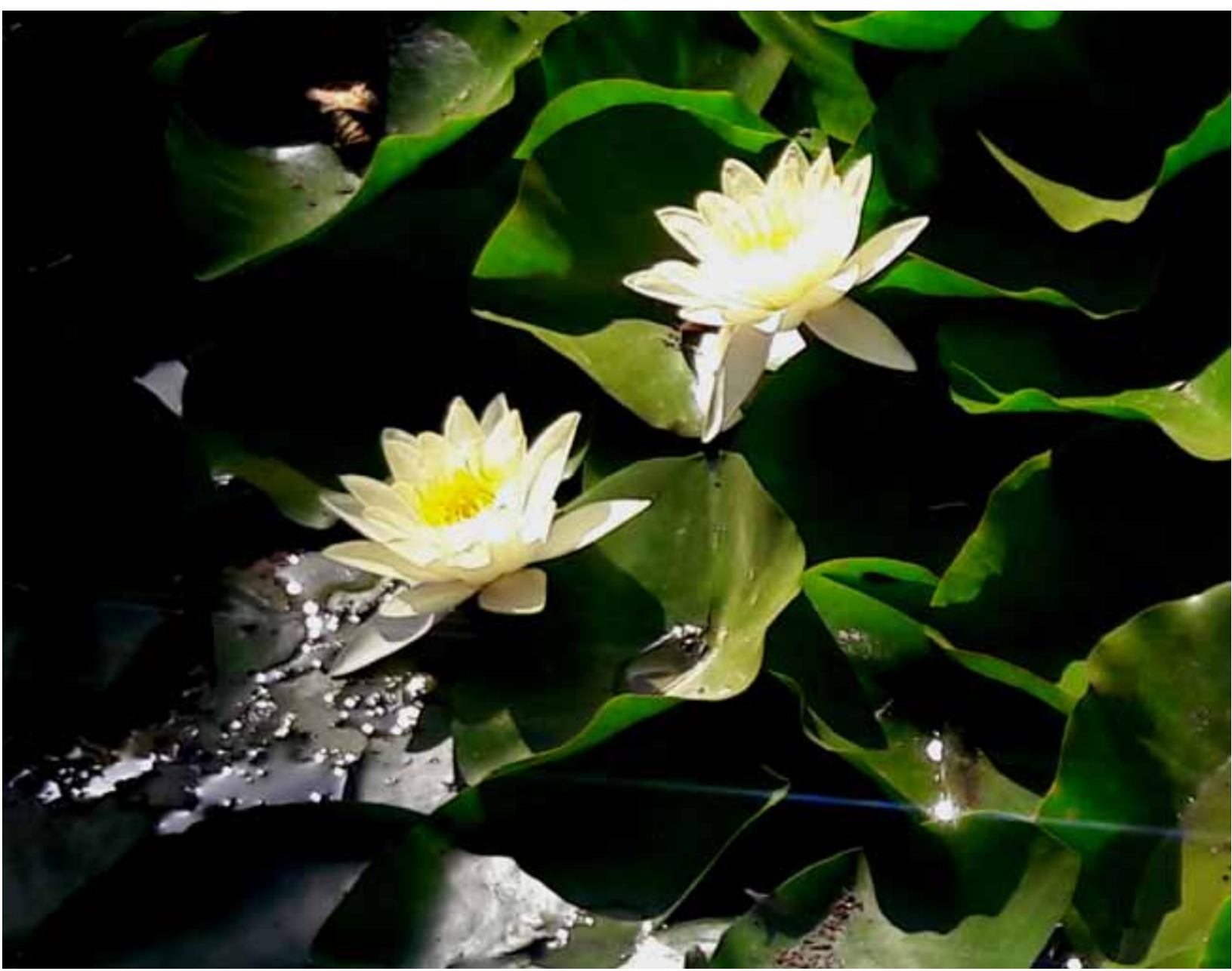

Cortesía de Silvina Spina 